\title{
Incidence of malignancy in patients with common variable immunodeficiency according to therapeutic delay: an Italian retrospective, monocentric cohort study
}

\author{
Veronica Pedini ${ }^{1,2}$, Jacopo Umberto Verga ${ }^{3}$, Irene Terrenato ${ }^{4}$, Denise Menghini ${ }^{1}$, Cristina Mezzanotte ${ }^{1}$ \\ and Maria Giovanna Danieli ${ }^{{ }^{*}}$
}

\begin{abstract}
Background: Common variable immunodeficiency (CVID) is the most common symptomatic primary immunodeficiency and has a broad spectrum of clinical manifestations. Among non-infectious complications, an increased incidence of malignancies may have a special relevance for survival, but little is known about treatment efficacy on malignant complications.
\end{abstract}

Methods: This was a monocenter retrospective study on CVID patients, designed to provide preliminary data for the investigation of the possible link between therapeutic delay and tumor incidence.

Results: A total of 67 CVID subjects were included. The median diagnostic delay was 7.5 years (range: 0-63 years), and the median therapeutic delay was 8.5 years (range: 0-67 years). Malignancies were diagnosed in 18 (27\%) patients. Eight out of 18 (44\%) patients with a malignancy had lymphoma. Patients who developed a malignancy showed a longer therapeutic delay in comparison to patients with no malignancy, although no statistical significance was achieved (11 years vs 8 years, respectively, $p=0.424$ ). We observed a lower frequency of malignancy in CVID patients with reduced therapeutic delay compared with patients with therapeutic delay $\geq 10$ years. With a therapeutic delay of $>1$ year, $74 \%$ had no tumor, and $25 \%$ had a tumor; with a therapeutic delay of $>10$ years, $65 \%$ had no tumor and $35 \%$ had a malignancy. Among patients who had no malignancy, $64 \%$ had a therapeutic delay of $<10$ years, and $36 \%$ had a therapeutic delay of $\geq 10$ years. Among patients with malignancy, $47 \%$ of subjects had a therapeutic delay $<10$ years, and $53 \%$ a therapeutic delay $\geq 10$ years.

Conclusions: The observation of clinical characteristics of our patients with CVID may suggest that an early institution of $\mathrm{gG}$ replacement therapy could be of benefit for the prevention of malignant complications.

Name of the registry: Comitato Etico Regionale delle Marche. Trial registration number: 1505. Date of registration: 27/10/2016, Retrospectively registered URL of trial registry record: http://www.ospedaliriuniti.marche.it/portale/archi vio13_cerm-ancona_0_446_1.html. The trial was not registered before the first participant was enrolled

Keywords: Common variable immunodeficiency, Diagnostic delay, Therapeutic delay, Ig replacement therapy

*Correspondence: m.g.danieli@univpm.it

${ }^{1}$ Medical Clinic, United Hospitals and DISCLIMO, Polytechnic University of Marche, Ancona, Italy

Full list of author information is available at the end of the article

\section{Introduction}

Common variable immunodeficiency (CVID) is the most common symptomatic primary immunodeficiency in adults and is characterized by reduced serum levels 
of immunoglobulin (Ig) G, IgA and/or IgM. Specific antibody production is reduced, or absent and several immune cell defects have been recognized in CVID patients $[1,2]$.

CVID has a broad spectrum of clinical manifestations, which may be gathered into two main phenotypes, possibly needing differential management: one group of patients are predominantly affected by recurrent infections and a second group of patients, who have a poorer prognosis, have autoimmune/inflammatory manifestations [3-5].

Among non-infectious complications, an increased incidence of malignancies may have a special relevance for survival. A higher frequency of malignancy in CVID patients compared with the general population, with a 5-12-fold risk increase, has been reported [6, 7]. In a recent meta-analysis, the prevalence of malignancy in CVID patients was found to be $8.6 \%$, with a prevalence of lymphoma of $4.1 \%$ [8]. The clinical spectrum of noninfectious complications has been recently described in a cohort of 623 patients with CVID in the USA [2]. In this cohort, lymphomas had an incidence of $6.7 \%$, and other tumors had an incidence of 6.4\%. Lymphomas (all were B cell type except one subject) were diagnosed more often in females than in males $(p=0.036)$ [2]. Remarkably, as reported by Odnoletkova et al., the prevalence of solid tumors in 2700 European patients with CVID significantly correlates with diagnostic delay (HR: 1.08, 95\% CI 1.04-1.11; p < 0.0001) [9]. In particular, each year of diagnostic delay was associated with an increase of the risk of solid tumors by $8 \%$.

While IgG replacement therapy has effectively improved the prognosis of CVID patients with prevalent infectious diseases, to our knowledge no study has tried to correlate delayed institution of replacement therapy (hence, therapeutic delay) with the onset of neoplasms [2, 10]. To address this issue, the relationship between therapeutic delay and cancer incidence should be studied. The clinical pattern, risk factors and the pathophysiological mechanisms of malignancies in subjects with CVID need to be thoroughly described, to prompt investigation on the possible preventive role of therapy.

This monocenter retrospective study on CVID patients, was designed to provide preliminary data for the investigation of the possible link between therapeutic delay and tumor incidence.

\section{Patients and methods}

This was a monocenter retrospective study, carried out at Medical Clinic, Ancona, a referral university hospital in the centre of Italy. All patients' data were recorded and analysed from our dedicated database. All patients previously gave informed consent to data collection and publication (Prot. N. 20160561 OR, 27/10/2016; n. $871 \mathrm{DG}, 7 / 12 / 2016)$. This specific study was further submitted for the approval of the local Ethical Committee (n. 1505).

Patients with CVID, aged $\geq 18$ years, and followed-up for $\geq 1$ year were included in the study. Patients were diagnosed with CVID according to the revised European Society for Immunodeficiency (ESID) criteria, and/or according to the International Consensus Document (ICON) criteria, for cases preceding ESID 2019 revision $[1,11]$. They were treated and followed-up according to the current clinical practice relative to the time of their management. The onset of CVID was defined by the presence of the first finding related to the disease.

Clinical phenotypes were defined according to Chapel et al. [12]. The diagnosis of GLILD was based on clinical features, ventilatory restrictive pattern at lung spirometry, high-resolution computed tomographic imaging of the chest [13] and exclusion of other conditions. Even if our patients did not perform lung biopsy, this is not strictly necessary in specific clinical conditions [14]. Moreover, our patients underwent lymph node, cutaneous and liver biopsies documenting the presence of non-caseating granulomatous lesions. Enteropathy was detected by colonoscopy and subsequent histological examination.

\section{Assessments, follow-up and treatment}

According to our practice, at first examination, we collected a full anamnesis for each patient, comprising complete past medical history, physiological anamnesis, familiarity for immunodeficiencies and autoimmune diseases and current medical situation, consanguinity among parents and grandparents. We also collected the interval among first symptoms and diagnosis (diagnostic delay) and start of treatment (therapeutic delay). Blood tests at the first visit included both routine ones and more specific examinations aimed at confirming the diagnosis of CVID, excluding a secondary immunodeficiency and predicting possible complications.

Clinical follow-up was performed every 3-6 months. At each control, all patients underwent a complete physical examination, including superficial lymph nodes and spleen evaluation. Patients were tested for Ig levels and routine analysis every 3-6 months and every 12-24 months for exclusion of emerging causes of secondary immunodeficiency.

In order to assess organ involvement, we performed at diagnosis: high-resolution chest $\mathrm{CT}$ scan and pulmonary function tests with $\mathrm{CO}$ diffusion capacity; esophageal gastroscopy with biopsy; abdominal ultrasound; 
oncological screening as for general population; other examinations (colonoscopy, thyroid ultrasound, etc.) in selected cases. These analyses were performed at follow-up as needed. All patients repeated at least annually pulmonary function tests, abdominal ultrasound and oncological screening.

As concerns Ig replacement therapy, patients received intravenous immunoglobulin (IVIg) or $20 \%$ subcutaneous immunoglobulin (SCIg, Hizentra ${ }^{\circledR}$ CSL Behring, King of Prussia, USA; starting from 2008, in the first years the $16 \%$ formulation was adopted) or facilitated-SCIg (fSCIg, Hyqvia ${ }^{\circledR}$, Takeda, Japan, starting from 2014) at the monthly dose of $0.4-0.6 \mathrm{~g} / \mathrm{kg}$. Side effects were registered during treatment in a diary provided to patients. Dose adjustments were based on infective recurrences and serum IgG levels. Goals of treatment were as follows: $\leq 2$ infectious episodes/years and serum IgG levels $\geq 700 \mathrm{mg} / \mathrm{dL}$. In case of recurrent infections despite appropriate serum IgG levels, antibiotic prophylaxis with macrolides was adopted (azithromycin $250 \mathrm{mg}$ /day for 3 days weekly).

The aim of Ig replacement therapy is that to prevent infectious episodes since there is not a universally accepted serum IgG trough level $[15,16]$. In our clinical practice, the target is a serum IgG levels around 700$800 \mathrm{mg} / \mathrm{dL}$ with differences between the Centers even within the same country $[17,18]$.

\section{Objectives}

The primary objective was to evaluate the frequency of therapeutic delay, expressed as years in CVID patients with or without malignant complications. Information on diagnostic delay was also collected.

Diagnostic delay was defined as the time between the occurrence of the first CVID symptom (reported in clinical charts or estimated on patient's memories) and the effective CVID diagnosis; and therapeutic delay as the time between the occurrence of the first symptom (reported in clinical charts or estimated on patient's memories) and the initiation of IgG replacement therapy. Data were censored on 31 January 2020.

\section{Statistical analysis}

Descriptive statistics were calculated for all variables of interest. Categorical data were presented as frequencies and percentage values, continuous variables as median values and their relative range. The Mann-Whitney nonparametric test and the Pearson's Chi square test, when appropriate, were applied to test potential differences between groups. $\mathrm{p}<0.05$ was considered statistically significant. All analyses were carried out with SPSS (SPSS version 21.0, IBM, Armonk, NY, USA).

\section{Results}

\section{Subjects and follow-up}

A total of 67 subjects were included in the study, with a CVID diagnosis received between 1983 and 2019. Patients were followed-up at this center since January 2008 with a median follow-up of 50 months (range: 0-183 months). Main patients' demographic and clinical characteristics at diagnosis are reported in Table 1. Median age at first CVID symptom occurrence was 30 years (range: 10-75 years), while median age at CVID diagnosis was 50 years (range: 16-79 years). At the onset, CVID patients presented with sinopulmonary tract infections (isolated, $\mathrm{n}=58$; associated with other features, $\mathrm{n}=5$ ), autoimmune cytopenia $(n=5)$ enteropathy $(n=2)$ and neoplasia $(\mathrm{n}=2)$. GLILD was diagnosed in six patients. Lymph node $(\mathrm{n}=3)$, cutaneous $(\mathrm{n}=1)$ and liver $(\mathrm{n}=2)$ biopsies were performed to document the presence of non-caseating granulomatous lesions. As for enteropathy, 11 patients presented collagenous colitis $(n=2)$ Crohnlike disease $(n=2)$ and colitis $(n=7)$.

A median diagnostic delay of 7.5 years (range: 0-63 years) was reported. At the time of data analysis, seven patients had died. Median serum Ig levels at diagnosis were IgG: $347 \mathrm{mg} / \mathrm{dL}$; IgA: $31 \mathrm{mg} / \mathrm{dL}$; and IgM: $28 \mathrm{mg} / \mathrm{dL}$.

During our follow-up, several non-infectious complications occurred (Table 2). Organ-specific and systemic autoimmune manifestations were present in $30(44 \%)$ patients. In all series, cytopenia was detected in $20(30 \%)$ patients, among which 12 had immune thrombocytopenic purpura (ITP), and three had autoimmune hemolytic anemia. Granulomatous disease in any site occurred in 12 (18\%) patients, among which six had granulomatous and lymphocytic interstitial lung disease, and eight had more than one organ involvement (lymph nodes, liver, skin). A CVIDassociated enteropathy was found in 11 (16\%) subjects,

Table 1 Characteristics of the patients at baseline

\begin{tabular}{ll}
\hline Criteria & $\mathbf{n ~ ( \% ) * ~}$ \\
\hline No of patients & $67(100)$ \\
Gender & \\
Male & $21(31)$ \\
Female & $46(69)$ \\
Age at disease onset (years); median (min-max) & $30(10-75)$ \\
Age at diagnosis (years); median (min-max) & $50(16-79)$ \\
Diagnostic delay (years); median (min-max) & $7.5(0-63)$ \\
Therapeutic delay (years); median (min-max) & $8.5(0-67)$ \\
\hline
\end{tabular}

*Variables are represented by frequencies and percentage values, unless otherwise specified 
Table 2 Clinical features and kind of treatment at last follow-up visit in 67 common variable immunodeficiency patients

\begin{tabular}{|c|c|}
\hline Criteria & n (\%) \\
\hline Non complicated patients & $21(31)$ \\
\hline \multicolumn{2}{|l|}{ Cytopenia } \\
\hline Autoimmune hemolytic anemia & $3(4)$ \\
\hline Neutropenia & $3(4)$ \\
\hline Immune thrombocytopenia & $12(18)$ \\
\hline \multicolumn{2}{|l|}{ Autoimmunity } \\
\hline Yes & $30(44)$ \\
\hline \multicolumn{2}{|l|}{ Granulomatosis } \\
\hline Yes & $12(18)$ \\
\hline Granulomatous and lymphocytic interstitial lung disease & $6(9)$ \\
\hline Granulomatosis in only 1 site & $3(4)$ \\
\hline Granulomatosis in $>1$ site & $9(13)$ \\
\hline \multicolumn{2}{|l|}{ CVID-associated enteropathy } \\
\hline Yes & $11(16)$ \\
\hline Helicobacter pylori positivity & $18(26)$ \\
\hline \multicolumn{2}{|l|}{ Allergies } \\
\hline Overall & $15(22)$ \\
\hline Drugs & $12(18)$ \\
\hline Splenectomy & $6(9)$ \\
\hline \multicolumn{2}{|l|}{ Therapy } \\
\hline 20\% SClg replacement therapy & $28(42)$ \\
\hline Facilitated SClg replacement therapy & $17(25)$ \\
\hline IVIg replacement therapy & $26(38)$ \\
\hline
\end{tabular}

CVID: Common variable immunodeficiency; IVlg: intravenous immunoglobulin; SClg: subcutaneous immunoglobulin

while Helicobacter pylori infection was demonstrated in $18(27 \%)$ patients.

\section{Therapeutic delay}

The median therapeutic delay was 8.5 (range: 0-67 years). A total of $64 / 67$ patients received a therapy for CVID; subcutaneous IgG replacement was administered to 28 patients, facilitated subcutaneous Ig therapy was used in 17 cases, and intravenous administration was used in 26 patients. Due to previous reaction to IVIg, three patients received antibiotic prophylaxis with azithromycin.

\section{Malignancies}

An oncologic disease was diagnosed in 18 (27\%) patients, two of which had two distinct primary lesions (Table 3).

Comparing patients who had malignant complications either before or during follow-up and those without tumors, the median age at CVID onset (29 years, range: $10-75$ vs 30 years, range: $10-75)$, the median age at CVID diagnosis (52 years, range: $20-79$ vs 46 years, range: $16-78)$, and Ig levels at CVID diagnosis (316 mg/
Table 3 Characteristics of malignancies in common variable immunodeficiency patients

\begin{tabular}{lc}
\hline Criteria & $\mathbf{n ~ ( \% ) ^ { * }}$ \\
\hline Cancer & \\
No & $49(73)$ \\
Yes & $18(27)$ \\
$\quad$ Lymphoma & $8(44)$ \\
$\quad$ Solid tumor & $10(56)$ \\
Age at cancer diagnosis (1) (years); median (min-max) & $53(21-85)$ \\
Age at cancer diagnosis (2) (years); median (min-max) & $76(41-85)$ \\
Time from first cancer diagnosis to CVID (months); & $159(10-692)$ \\
$\quad$ median (min-max) &
\end{tabular}

CVID: Common variable immunodeficiency

*Variables are represented by frequencies and percentage values, unless otherwise specified

$\mathrm{dL}$ vs $388 \mathrm{mg} / \mathrm{dL}$ ) were similar, at the time of data analysis (Table 4).

Overall, eight malignancies were diagnosed prior to CVID (mean time before diagnosis: 54 months), while the others occurred during the follow-up (mean time after CVID diagnosis: 160 months).

At diagnosis of malignancy (data available for 12 patients), the median level of IgG was $792 \mathrm{mg} / \mathrm{dL}$ (range: 506-1350); one patient was not receiving replacement therapy due to a previous severe adverse reaction. A malignant complication was the death cause for four out of seven dead patients.

Median age at the time of the first malignancy diagnosis was 53 (range: $21-85$ ) years; while the median age at the time of a second malignancy diagnosis was 76 (range: 41-85) years (Table 3). Patients diagnosed with a malignancy before CVID, compared with those with tumors occurred after the CVID diagnosis, had a lower mean age at tumor diagnosis (43 vs 61 years, respectively), and a longer diagnostic delay for CVID (130 vs 49 months, respectively). Hypogammaglobulinemia had been found before the tumor occurrence in all eight patients with a malignancy preceding CVID, but not further investigated.

A total of eight out of 18 (44\%) patients with an oncologic disease had a lymphoma (overall incidence: 12\%): one mycosis fungoides, one large granular lymphocytic leukemia and six B-cell lymphomas (two were gastrointestinal lymphomas). No patient had both a lymphoproliferative malignancy and a solid tumor. Four lymphoproliferative malignancies occurred prior to the CVID diagnosis, and four during the follow-up, 4, 6, 7 and 21 years after the CVID diagnosis, respectively.

Ten patients had solid tumors and of these, two developed a second solid tumor. The 12 solid tumor types 
Table 4 Comparison between cancer/no cancer patients and demographic/clinical characteristics

\begin{tabular}{|c|c|c|c|}
\hline \multirow[t]{2}{*}{ Criteria } & \multicolumn{2}{|l|}{ Cancer } & \multirow[t]{2}{*}{ p-value } \\
\hline & Yes $(n=18)$ & No $(n=49)$ & \\
\hline Age at CVID onset; median (min-max) & $29(10-75)$ & $30(10-75)$ & 0.769 \\
\hline Age at CVID diagnosis; median (min-max) & $52(20-79)$ & $46(16-78)$ & 0.449 \\
\hline Autoimmunity: Yes (n) & 11 & 19 & 0.103 \\
\hline ITP:Yes (n) & 5 & 7 & 0.202 \\
\hline Granulomatosis: Yes (n) & 3 & 9 & 0.872 \\
\hline CVID-associated enteropathy: Yes (n) & 2 & 9 & 0.477 \\
\hline
\end{tabular}

CVID: Common variable immunodeficiency; ITP: Immune thrombocytopenic purpura; CVID onset was defined as the occurrence of the first findings related to CVID (see "Patients and methods" for details)

were: three melanoma, two thyroid cancers, one gastric schwannoma, two breast cancer, one pancreas cancer, two gastric cancers and one bladder cancer.

While the overall incidence of autoimmune disease in our case series was $44 \%$, the condition was not equally distributed in patients with and without malignancy; in the absence of oncological complications the incidence was 38\% (19/49 patients), but in the presence of malignancy it was $61 \%$ (11/18 patients). Most of the autoimmune diseases were represented by ITP, with an incidence of $14 \%$ and $27 \%$ (7/49 patients without malignancy, and $5 / 18$ patients with malignancy).

\section{Malignancy and therapeutic delay}

The median therapeutic delay was 11 years in patients with malignancy vs 8 years in those without malignant complication $(p=0.424)$. Although a statistical significance was not achieved these results suggest a possible link between the therapeutic delay and tumor incidence (Table 5). Furthermore, among patients who had no malignancy at end of follow-up, $64 \%$ had a therapeutic delay of $<10$ years, and $36 \%$ had a therapeutic delay of $\geq 10$ years. Conversely, among patients with one or more malignancies, a therapeutic delay of $<10$ years was recorded only for $47 \%$ of subjects, and a therapeutic delay of $\geq 10$ years for $53 \%$ of subjects (Fig. 1a).

Similar observations were made for the diagnostic delay (Table 5 and Fig. 1b). The median diagnostic delay was longer in patients with malignancy compared to those who had no oncologic complication (11 years vs 5 years, respectively, $\mathrm{p}=0.579)$. Among patients without malignant complications a diagnostic delay $\leq 10$ years was reported in $69 \%$ of patients, while a diagnostic delay $>10$ years was observed in $31 \%$ of patients without tumor; differently, patients with malignant complications had the same frequency of diagnostic delay $\leq 10$ years and $>10$ years. Among the 47 patients with a diagnostic delay of $<10$ years, $74 \%$ had no malignancy, and $25 \%$ had
Table 5 Comparison between cancer/no cancer patients and therapeutic and diagnostic delays

\begin{tabular}{llll}
\hline & \multicolumn{2}{c}{ Cancer } & p-value \\
\cline { 2 - 3 } & Yes $(\mathbf{n}=\mathbf{1 8})$ & No $(\mathbf{n = 4 9 )}$ & \\
\hline $\begin{array}{c}\text { Therapeutic delay } \\
\begin{array}{c}\text { (years); median } \\
(\text { min-max) }\end{array}\end{array}$ & $11(0-67)$ & $8(0-50)$ & 0.424 \\
$\begin{array}{c}\text { Diagnostic delay } \\
\text { (years); median } \\
(\text { min-max) }\end{array}$ & $11(0-63)$ & $5(0-9)$ & 0.579 \\
\hline
\end{tabular}

*Times were calculated from the detected or estimated disease onset data

it; among the 24 patients with diagnostic delay $>10$ years, $62 \%$ had not an oncologic disease and $37 \%$ had it.

A comparison between cancer/noncancer patients and diagnostic and therapeutic delays categorized by different cut-off (sensitivity analysis) is reported as Additional file 1.

\section{Discussion}

Mechanisms involved in the high susceptibility to malignancy of CVID patients are not totally clarified. Several mechanisms have been hypothesized to be involved, like genetic predisposition and genetic instability; persistent activation, and proliferation of the lymphoid cells during infections; decreased clearance of oncogenic viruses and bacterial infections.

In patients with CVID, the impaired immune response to infections is responsible for the chronic antigen stimulation, the chronic inflammation and the increased survival and proliferation of premalignant and malignant cells. It is, thus, interesting hypothesize that Ig replacement therapy with the consequent reduction of infections and related complications may decrease the risk of malignancy linked to the impaired immune response.

We have already described the potential antitumor activity of immunoglobulin, thanks to its 


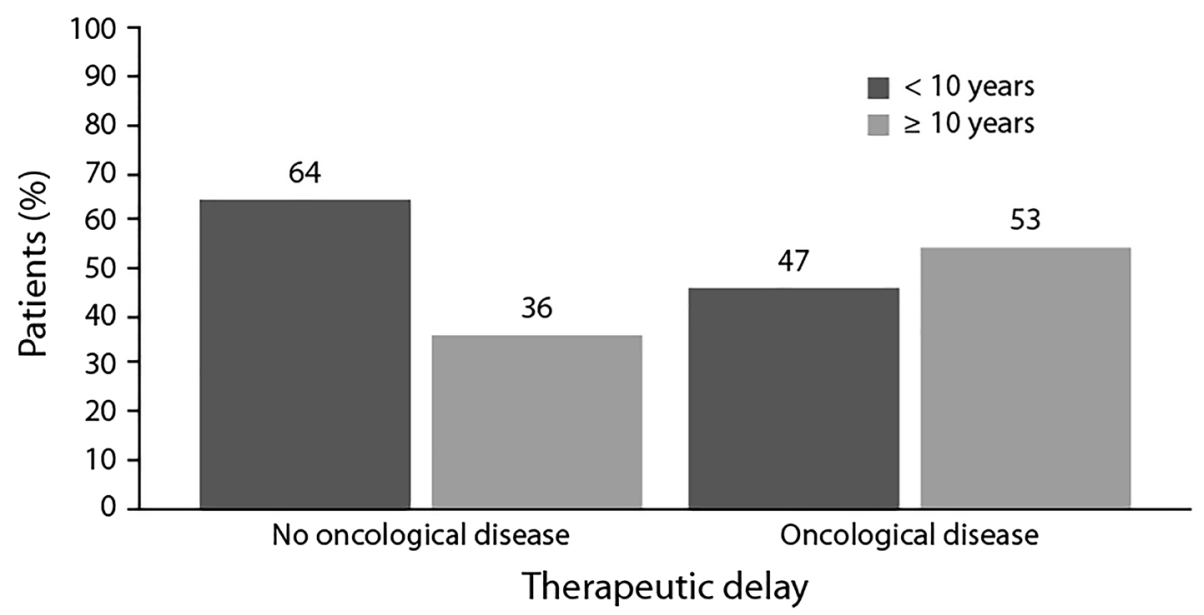

Fig. 1 Incidence of therapeutic delays either $\leq 10$ years or $>10$ years in patients with malignancy or without malignant complications of CVID

immunomodulatory role due to its action in the adaptive immunity and in the maintenance of immune homeostasis (activation of FCGR; stimulation and NK cells cytotoxic activity on tumor cells; increased expression of pro-apoptotic molecules; reduction of tumor spread by Anti-VEGF Ab and reduced expression of metalloproteinases and anti-RGD Ab, etc.) [19]. However, to our knowledge, the correlation between delayed institution of Ig replacement therapy and the onset of cancer disease has never been investigated to date.

This retrospective observational study describes the clinical features of malignancies in 67 patients with CVID, followed-up for over 10 years in a single specialized center in Italy. The information obtained from this cohort of patients should be useful for further investigation on the effect of IgG replacement treatment on incidence and progression of tumors in CVID patients. We acknowledge that this study has several limitations, including its retrospective design, and the limited number of patients-however, in line with the rarity of the disease-which precluded us the possibility of a more refined statistical analysis taking into account all the variables. In addition, this was not an interventional study and can only suggest the relevance of the diagnostic and therapeutic delay. Last, in a limited number of cases $(n=5)$, information on therapeutic delay was collected based on patients' memories We however believe that a careful description of a homogeneously followed-up population may nevertheless be interesting for researchers.

As expected, the incidence of malignancy and the proportion of lymphomas were high in our group of patients compared to the general population $[6,7]$. In our group, $27 \%$ of patients had a malignant disease, with an incidence of lymphoma of $12 \%$; so, we found higher incidences than those found by the meta-analysis by Kiaee et al. [8], but in line with other reports [20,21].

Lymphomas were the most frequent malignancy, representing $40.5 \%$ of cancers in the meta-analysis by Kiaee et al., and $53.4 \%$ in the study by Resnick et al. [5, 8]. Our case series agreed with these data, as lymphomas were $44 \%$ of malignancies. Tumors were a very relevant cause of death in our cohort (four out of seven deaths), more than infections (two cases).

In our series, we documented a higher incidence of autoimmune cytopenia, in particular ITP, in patients who developed neoplasia than in those without malignant complications. It is possible that we observed very selected cases, with serious manifestations, as we operated in a tertiary care Centre. However, this data agrees with findings by Kralickova et al., who identified ITP as a potential risk factor for cancer development in CVID patients [22]. In addition, the observation further suggests that the immune dysregulation underlying CVID may promote malignant occurrence [22].

The main result of our study is that the median diagnostic delay was longer in patients with malignancy compared to those who had no oncologic complication and similar data were observed for the median therapeutic delay; although a statistical significance was not achieved probably due to the intrinsic limit of the study, that is limited sample size as a consequence of a rare condition, these results suggest a possible link between the diagnostic and therapeutic delay and tumor incidence. Furthermore, if these data were confirmed by a larger study, the information would support the hypothesis that the IgG replacement 
therapy could have a role in cancer prevention in CVID patients. Almost all the patients in this series received IgG replacement therapy, mainly through the subcutaneous route, but time of initiation was greatly variable, and a long period without treatment seems to impact on cancer risk.

Similarly, the diagnostic delay for CVID was found to be longer in patients with tumor onset previous to CVID diagnosis compared with those who had tumors after the CVID diagnosis. It is possible that malignancy was an early manifestation of undiagnosed CVID in these former subjects. Patients affected by the CVID phenotype characterized by a low incidence of infections and a prevalent incidence of noninfectious manifestations are at an increased risk of misdiagnosis, as clinician attention is not easily raised toward immune impairment in the absence of recurrent infection. In our case series, all patients with a tumor occurred before CVID diagnosis had been found with hypogammaglobulinemia even before tumor occurrence, but this clue had not been interpreted as an early symptom of CVID and it was not further investigated.

In conclusion, although with all the limitations of any retrospective study, the observation of clinical characteristics of our patients with CVID may suggest that an early institution of IgG replacement therapy could be of benefit for the prevention of malignant complications.

\section{Supplementary information}

Supplementary information accompanies this paper at https://doi. org/10.1186/s13223-020-00451-z.

Additional file 1: Table S1. Comparison between cancer/no cancer patients and diagnostic and therapeutic delays categorized by different cut-off.

\section{Abbreviations}

CVID: Common variable immunodeficiency; Ig: Immunoglobulin; ESID: European Society for Immunodeficiency; ICON: International Consensus Document; ITP: Immune thrombocytopenic purpura.

\section{Acknowledgements}

Editorial assistance was provided by Laura Brogelli, MD, Luca Giacomelli, PhD, and Aashni Shah, of Polistudium srl (Milan, Italy); this assistance was supported by Csl Behring Spa (Milan, Italy).

\section{Authors' contributions}

VP, DM, CM and MGD contributed to the diagnosis, treatment and follow-up of patients and interpreted analysed data; JUV collected data; IT analysed data. VP and MGD wrote and revised critically the manuscript. All authors read and approved the final manuscript.

\section{Funding}

This study was supported by internal funds.

\section{Availability of data and materials}

The datasets used and/or analysed during the current study are available from the corresponding author on reasonable request.

\section{Ethics approval and consent to participate}

All patients gave informed consent to data collection and publication (Prot. N. 20160561 OR, 27/10/2016; n. 871 DG, 7/12/2016). This specific study was further submitted for the approval of the local Ethical Committee (n. 1505).

\section{Consent for publication}

All patients previously gave informed consent publication (Prot. N. 20160561 OR, 27/10/2016; n. 871 DG, 7/12/2016).

\section{Competing interests}

The authors declare that they have no competing interests.

\section{Author details}

${ }^{1}$ Medical Clinic, United Hospitals and DISCLIMO, Polytechnic University of Marche, Ancona, Italy. ${ }^{2}$ Medicine Departement, Destra Secchia Hospital, Pieve di Coriano, ASST Mantova, Mantua, Italy. ${ }^{3}$ Molecular and Applied Biology, Polytechnic University of Marche, Ancona, Italy. ${ }^{4}$ Biostatistic and Bioinformatic Unit, Scientific Direction, IRCCS Regina Elena National Cancer Institute, Rome, Italy.

Received: 17 April 2020 Accepted: 15 June 2020

Published online: 26 June 2020

\section{References}

1. Bonilla FA, Barlan I, Chapel H, Costa-Carvalho BT, Cunningham-Rundles C, de la Morena MT, et al. International Consensus Document (ICON): common variable immunodeficiency disorders. J Allergy Clin Immunol Pract. 2016;4(1):38-59. https://doi.org/10.1016/j.jaip.2015.07.025.

2. Ho HE, Cunningham-Rundles C. Non-infectious complications of common variable immunodeficiency: updated clinical spectrum, sequelae, and insights to pathogenesis. Front Immunol. 2020;11:149. https://doi.org/10.3389/fimmu.2020.00149.

3. Perez EE, Orange JS, Bonilla F, Chinen J, Chinn IK, Dorsey M, et al. Update on the use of immunoglobulin in human disease: a review of evidence. J Allergy Clin Immunol. 2017;139:S1-46. https://doi.org/10.1016/j. jaci.2016.09.023.

4. Cunningham-Rundles C, Bodian C. Common variable immunodeficiency: clinical and immunological features of 248 patients. Clin Immunol. 1999;92:34-48. https://doi.org/10.1006/clim.1999.4725.

5. Resnick ES, Moshier EL, Godbold JH, Cunningham-Rundles C. Morbidity and mortality in common variable immune deficiency over 4 decades. Blood. 2012;119:1650-7. https://doi.org/10.1182/blood-2011-09-377945.

6. Mellemkjaer L, Hammarstrom L, Andersen V, Yuen J, Heilmann C, Barington $T$, et al. Cancer risk among patients with IgA deficiency or common variable immunodeficiency and their relatives: a combined Danish and Swedish study. Clin Exp Immunol. 2002;130(3):495-500.

7. Vajdic CM, Mao L, van Leeuwen MT, Kirkpatrick P, Grulich AE, Riminton $\mathrm{S}$. Are antibody deficiency disorders associated with a narrower range of cancers than other forms of immunodeficiency? Blood. 2010;116(8):1228-34

8. Kiaee F, Azizi G, Rafiemanesh H, Zainaldain H, Sadaat Rizvi F, Alizadeh M, et al. Malignancy in common variable immunodeficiency: a systematic review and meta-analysis. Expert Rev Clin Immunol. 2019;15(10):1105-13. https://doi.org/10.1080/1744666X.2019.1658523.

9. Odnoletkova I, Kindle G, Quinti I, Grimbacher B, Knerr V, Gathmann B, et al. The burden of common variable immunodeficiency disorders: a retrospective analysis of the European Society for Immunodeficiency (ESID) registry data. Orphanet J Rare Dis. 2018;13(1):201. https://doi. org/10.1186/s13023-018-0941-0.

10. Kaveri SV, Maddur MS, Hedge P, Lacroix-Desmazes S, Bayry J. Intravenous immunoglobulins in immunodeficiencies: more than mere replacement therapy. Clin Exp Immunol. 2011;164(Suppl 2):2-5. https://doi.org/10.111 1/j.1365-2249.2011.04387.x. 
11. European Society for immunodeficiencies (ESID). Common variable immunodeficiency diagnostic criteria. https://esid.org/Working-Parti es/Clinical-Working-Party/Resources/Diagnostic-criteria-for-PID2\#Q3. Accessed 17 Apr 2020.

12. Chapel H, Lucas M, Patel S, Lee M, Cunningham-Rundles C, Resnick E, et al. Confirmation and improvement of criteria for clinical phenotyping in common variable immunodeficiency disorders in replicate cohorts. J Allergy Clin Immunol. 2012;130(5):1197-8. https://doi.org/10.1016/j. jaci.2012.05.046.

13. Cinetto F, Scarpa R, Rattazzi M, Agostini C. The broad spectrum of lung diseases in primary antibody deficiencies. Eur Respir Rev. 2018;27:180019. https://doi.org/10.1183/16000617.0019-2018.

14. Raghu G, Collard HR, Egan JJ, Martinez FJ, Behr J, Brown KK, et al. An official ATS/ERS/JRS/ALAT statement: idiopathic pulmonary fibrosis: evidence-based guidelines for diagnosis and management. Am J Respir Crit Care Med. 2011;183:788-824. https://doi.org/10.1164/rccm.2009040GL.

15. Lucas M, Lee M, Lortan J, Lopez-Granados E, Misbah S, Chapel H. Infection outcomes in patients with common variable immunodeficiency disorders: relationship to immunoglobulin therapy over 22 years. J Allergy Clin Immunol. 2010;125(6):1354-1360.e4. https://doi.org/10.1016/j. jaci.2010.02.040.

16. Bonagura VR, Marchlewski R, Cox A, Rosenthal DW. Biologic lgG level in primary immunodeficiency disease: the lgG level that protects against recurrent infection. J Allergy Clin Immunol. 2008;122(1):210-2. https://doi. org/10.1016/j.jaci.2008.04.044.
17. Jolles $\mathrm{S}$. The variable in common variable immunodeficiency: a disease of complex phenotypes. J Allergy Clin Immunol Pract. 2013;1 (6):545-56. https://doi.org/10.1016/j.jaip.2013.09.015.

18. Jolles S. When to initiate immunoglobulin replacement therapy (IGRT) in antibody deficiency: a practical approach. Clin Exp Immunol. 2016;188:333-41. https://doi.org/10.1111/cei.12915.

19. Danieli MG, Gelardi C, Pedini V, Gabrielli A. Potential anti-tumor activity of intravenous and subcutaneous immunoglobulin. Isr Med Assoc J. 2018;20(12):782-3.

20. Pulvirenti F, Pecoraro A, Cinetto F, Milito C, Valente M, Santangeli E, et al. Gastric cancer is the leading cause of death in italian adult patients with common variable immunodeficiency. Front Immunol. 2018;9:2546. https ://doi.org/10.3389/fimmu.2018.02546.

21. Baloh C, Reddy A, Henson M, Prince K, Buckley R, Lugar P. 30-year review of pediatric- and adult-onset CVID: clinical correlates and prognostic indicators. J Clin Immunol. 2019;39(7):678-87. https://doi.org/10.1007/ s10875-019-00674-9.

22. Kralickova P, Milota T, Litzman J, Malkusova I, Jilek D, Petanova J, et al. CVID-Associated Tumors: czech Nationwide Study Focused on Epidemiology, Immunology, and Genetic Background in a Cohort of Patients With CVID. Front Immunol. 2019;9:3135.

\section{Publisher's Note}

Springer Nature remains neutral with regard to jurisdictional claims in published maps and institutional affiliations.
Ready to submit your research? Choose BMC and benefit from:

- fast, convenient online submission

- thorough peer review by experienced researchers in your field

- rapid publication on acceptance

- support for research data, including large and complex data types

- gold Open Access which fosters wider collaboration and increased citations

- maximum visibility for your research: over $100 \mathrm{M}$ website views per year

At BMC, research is always in progress.

Learn more biomedcentral.com/submissions 